\title{
Importance of preoperative health status and intraoperative factors in predicting adverse outcomes in geriatric patients undergoing major gastrointestinal surgery
}

Pralaya Khadka

Resident, Department of Surgery, TU Teaching Hospital, Maharajgunj, Kathmandu.

Email: pralayakhadka@gmail.com

\begin{abstract}
:
Introduction and objective: Perioperative management of elderly (aged 65 or older) surgical patients has become an essential component of surgical and anesthetic practice. Certain preoperative and intraoperative factors may predict adverse outcomes in such patients. The objective was to determine the prevalence and predictors of adverse postoperative outcomes in older surgical patients undergoing major gastrointestinal surgery.
\end{abstract}

Materials and Methods: Prospective study of consecutive elderly patients who underwent major gastrointestinal surgery between August 2015 and November 2015 in Tribhuwan University Teaching Hospital. Patients were followed up to 1 month after surgery.

Potential pre- and intra-operative risk factors were measured and evaluated for their association with the occurrence of predefined adverse outcomes. Multivariate logistic regression of predictors of postoperative outcomes were measured. Odds ratios (OR) with 95\% confidence interval (Cl) and two-sided p-values were reported.

Results:One hundred and thirty-three consecutive patients were studied. Overall, 89 (66.9\%) of patients developed one or more postoperative adverse outcomes and $6(4.51 \%)$ died during their postoperative period. Of all the adverse outcomes, Respiratory (38.3\%) were the leading cause of morbidity, followed by Gastrointestinal (32.3\%) and Cardiovascular (28.8\%) complications.

By multivariate logistic regression analysis, presence of comorbidites (OR 1.8, CI 1.1-2.8), low albumin (OR 2.71, $\mathrm{Cl}$ 0.1-6.6), tachycardia (OR 3.7, $\mathrm{Cl}$ 1.2-10.8) and hypotension (OR 8.9, $\mathrm{Cl}$ 1.1-17) were the significant predictors of postoperative adverse outcomes. The mean hospital stay was $7.4 \pm 3.8$ days.

Conclusion: Low albumin and presence of comorbidities, tachycardia and hypotension are significant predictors of adverse surgical outcomes. Age by itself is not associated with adverse outcomes.

Keywords: geriatric; outcomes; predictors; surgery 